LA W RENCE LIVERMORE N A T IO N A L LABORATORY

\title{
Shock Compressing Diamond to a Conducting Fluid
}

D. K. Bradley, J. H. Eggert, D. G. Hicks, P. M. Celliers, S. J. Moon, R. C. Cauble, G. W. Collins

July 30, 2004

Physical Review Letters 
This document was prepared as an account of work sponsored by an agency of the United States Government. Neither the United States Government nor the University of California nor any of their employees, makes any warranty, express or implied, or assumes any legal liability or responsibility for the accuracy, completeness, or usefulness of any information, apparatus, product, or process disclosed, or represents that its use would not infringe privately owned rights. Reference herein to any specific commercial product, process, or service by trade name, trademark, manufacturer, or otherwise, does not necessarily constitute or imply its endorsement, recommendation, or favoring by the United States Government or the University of California. The views and opinions of authors expressed herein do not necessarily state or reflect those of the United States Government or the University of California, and shall not be used for advertising or product endorsement purposes. 


\title{
Shock compressing diamond to a conducting fluid
}

D. K Bradley, J. H. Eggert, D. G. Hicks, P. M. Celliers, S. J. Moon, R. C. Cauble, and

G. W. Collins

Lawrence Livermore National Laboratory, Livermore, CA 94551

(Dated: April 7, 2004)

\begin{abstract}
Laser generated shock reflectance data show that diamond undergoes a continuous transition from optically absorbing to reflecting between Hugoniot pressures $600<\mathrm{P}_{\mathrm{H}}<1000 \mathrm{GPa}$. The data are consistent with diamond having a thermal population of carriers at $\mathrm{P}_{\mathrm{H}} \sim 600 \mathrm{GPa}$, undergoing band overlap metallization at $\mathrm{P}_{\mathrm{H}} \sim 1000 \mathrm{GPa}$ and melting at $800<\mathrm{P}_{\mathrm{H}}<1000 \mathrm{GPa}$. The results agree well with an equation of state model that predicts that elemental carbon remains solid throughout the interior of Neptune.
\end{abstract}

PACS numbers: 52.50.Lp, 62.50.+p, 64.70.Dv, 71.30.+h 
Carbon is a basic building block of life, a primary constituent of Uranus, Neptune $^{1,2}$, and white dwarfs, and is used in many technological applications. The unique properties of the diamond phase, including its extreme hardness and low compressibility have made possible static high-pressure research up to several hundred $\mathrm{GPa}^{3}$. However, the properties of diamond at higher pressures remain unknown. Does carbon transform from diamond to another crystallographic phase at ultra high pressure and is there a metallic solid phase $?^{4-6}$ What is the melting curve of diamond? ${ }^{5-11}$ Is the high-pressure liquid state conducting? ${ }^{12,13}$ What is the state of carbon in Uranus and Neptune? ${ }^{2}$ We report on measurements that show that diamond melts to a conducting fluid between 600 and $1000 \mathrm{Gpa}$ on the principal Hugoniot. At pressures just below the onset of melt, our analysis suggests that the band gap closes. These observations suggest that pure carbon in the deep interior of Neptune would be in an insulating solid phase.

The multiple hybridisations of carbon's valence electrons result in a complicated phase diagram. Although the region around the graphite-liquid melt line has been studied extensively by several authors ${ }^{14-16}$, (showing that liquid carbon, at temperatures just above the melt line is a semi-metal), experimental data in the high-pressure region around the diamond melt curve are sparse ${ }^{12}$. Figure 1 shows one high-pressure phase diagram, proposed by van Thiel and $\operatorname{Re}^{9}$ (VTR). Superimposed on the VTR diagram are several theoretical diamond Hugoniots and melting curves ${ }^{5,8,10,17}$, and an isentrope that lies close to predicted core conditions of Neptune ${ }^{18}$. As can be seen, for all but the VTR model the high-pressure part of the isentrope is predicted to lie in the molten phase. In addition to the melting transition, several authors ${ }^{4}, 5,{ }^{19}$ predict that diamond will transform to a metallic BC8 phase before melting. This predicted insulating to metallic-solid transition 
is incorporated into the phase diagram proposed by Kerley and Chhabildas $(\mathrm{KC})^{5}$ and accounts for the changes in slope along the KC Hugoniot (figure 1) at 430-500 GPa. In contrast, Grumbach \& Martin ${ }^{6}$ estimate that a BC8-diamond-liquid triple point occurs near $2000 \mathrm{GPa}$ and $4300 \mathrm{~K}$ - which is inaccessible to the principal (single shock) Hugoniot of diamond

Experiments were conducted using the $351 \mathrm{~nm}$ OMEGA laser system at the University of Rochester. Targets consisted of 100 or $500 \mu \mathrm{m}$ thick natural type 2a diamond flats oriented along the (110) axis and glued onto the thin side of an Al step. A low- $\mathrm{Z}$ plastic $(\mathrm{CH})$ ablator on the drive side of the $\mathrm{Al}$ pusher reduced the quantity of high energy x-rays produced at the ablation front that can preheat the target. A thin $(100 \mathrm{~nm})$ coating of $\mathrm{Al}$ on the laser-drive side of the $\mathrm{CH}$ ensured that the laser plasma originated away from the Al plate. ${ }^{20}$ The diamond surface was coated with an $\mathrm{Al}_{2} \mathrm{O}_{3}$ anti-reflection coating to minimize reflection of the probe beam. A typical target is shown in figure 2 . The drive laser produces a shock, which propagates through the pusher, and into the diamond sample. Steady shocks used for Hugoniot P- $\rho$ data were achieved with 3.5 ns flat top pulse shapes with incident laser intensities in the range of $1 \times 10^{13}$ to $2 \times 10^{14}$ $\mathrm{W} / \mathrm{cm}^{2}$. Decaying shocks, used for mapping shock reflectance over a continuous pressure range were produced with 1 ns flat top pulse shapes. Shock planarity (2\% over $300 \mu \mathrm{m}$ upon breakout from a $50 \mu \mathrm{m} \mathrm{Al}$ target) was achieved using continuous phase plates having a flat-top footprint of $650 \mu \mathrm{m}$.

A line VISAR (Velocity Interferometer System for Any Reflector) diagnostic ${ }^{21}$ operating at $532 \mathrm{~nm}$ measured the shock velocity in diamond, $U_{s}^{\text {diamond }}$ versus time by detecting the Doppler shift of light reflecting off the shock front. ${ }^{22}$ Laser light reflected 
from the target is imaged through an interferometer onto the slit of an optical streak camera. The resulting streaked image contains a series of fringes, whose phase is directly proportional to the velocity of the reflecting surface ${ }^{23}$. The image also contains onedimensional spatial information from the reflector.

In general, transparent materials undergo a series of similar optical responses, from transparent, to non-transparent (due to absorption or diffusive scattering), to reflecting with increasing shock pressure. At low pressures ( $\leq 100 \mathrm{GPa})$ shocked diamond is known to be transparent ${ }^{24}$ (reflecting surfaces can be observed with a VISAR through a shocked diamond window), and we have used it as a window material for 1064 nm light for shocks up to $120 \mathrm{GPa}$. At $\sim 300<\mathrm{P}<600 \mathrm{GPa}$, shocked diamond is nontransparent (the reflected intensity at the VISAR detector lacks any contribution from surfaces beyond the shock front) and any VISAR signal at $532 \mathrm{~nm}\left(\mathrm{R}_{\lambda=532 \mathrm{~nm}}\right)$ from a potentially reflecting shock is below our detection limit of $0.1-1 \%$. An example VISAR record for $\mathrm{P}=550-600 \mathrm{GPa}$ (as determined by simulation and average transit time through the diamond) is shown in figure 1 (inset a). Following the VISAR fringes in time (from left to right) we note that before the shock breaks out from the Al, steady unshifted fringes are seen. Since no fringe shift is detected until the shock wave reaches the surface of the thin $\mathrm{Al}$ step and breaks out into the diamond, we estimate the preheat in the Al to be below $600 \mathrm{~K}$. At $~ 5.5$ ns the shock breaks out into the diamond and the reflected signal drops to near our detection limit. At $\sim 6.5 \mathrm{~ns}$ the shock breaks out of the thick $\mathrm{Al}$ step, and the reflection from the $\mathrm{Al}$ surface rapidly vanishes. The loss of surface reflectivity is due to the rapid development of a density gradient when a surface without strength (fluid) unloads into vacuum ${ }^{25}$. At $\sim 10$ ns the shock front breaks out from the rear 
surface of the diamond and in this case a bright series of fringes (10-20\% reflective) return. This fringe recovery occurs when a material with strength (solid) unloads into a vacuum (the antireflection coating on a window is generally destroyed by a strong shock).

The VISAR image in figure $1 \mathrm{~b}$ (inset $\mathrm{b}$ ) is from diamond shocked to a high pressure of $\sim 1050 \mathrm{GPa}$, which is above the predicted melt pressure $P_{\text {melt }}$ for all the models. Strong fringes are seen when the shock breaks out from the thin Al step into the diamond at 2.4 ns. The discontinuity seen at this time is caused by the streak camera being unable to resolve the rapid change in phase ( $\sim 4$ fringes) as the VISAR probe starts to reflect off the rapidly moving shock front. The shock velocity in the diamond (confirmed by a second VISAR with a different sensitivity) ranged from $25 \mu \mathrm{m} / \mathrm{ns}$ just after breakout to $26.4 \mu \mathrm{m} / \mathrm{ns}$ after $3 \mathrm{~ns}$. The reflectivity from the shock, $R_{\lambda=532 n m} \sim 30 \%$, was calculated by comparing to the reflectivity of the thicker (bare) Al step, which was taken to be $85 \%{ }^{26}$. This value for $R_{\lambda=532 n m}$ in diamond is more than an order of magnitude higher than expected from a Fresnel reflection, and as is discussed below results from reflection off a conducting phase of carbon.

In order to probe the transition region between non-reflecting (non-conducting) and reflecting (conducting) phases, a temporally short (1 ns) laser pulse was used to launch a shock that decays with time continuously over a large pressure range. This technique allows a large pressure range to be sampled on a single shot, and minimizes random uncertainties in reflectance at different pressures. While the shot to shot uncertainty in reflectance can be as high as $30 \%$, the relative uncertainty within a single shot is much less. Figure 2 shows an experimental VISAR data record which spans from 
P 1500 GPa just after shock breakout into the diamond to 500 GPa near the end of the data record. After the shock enters the diamond, the normalized reflected probe intensity is at a high value and then decays to the background level. This observation is analysed to give a continuous record of $R_{\lambda=532 n m}$ from shock compressed diamond and $U_{s}^{\text {diamond }}$ from the fringe phase shift. While the shock front pressure decreases with time, the shock front is always on the Hugoniot. Although there is a $P-\rho-T$ gradient behind the shock front, over an optical depth $(\sim 40 \mathrm{~nm})$ the variations in $P-\rho-T$ are very small.

Figure 3 shows $R_{\lambda=532 n m}$ plotted versus $U_{s}^{\text {diamond }}$ for two shots. The shock front reflectance is constant at about $30 \%$ over a wide range of shock velocities above 24.5 $\mu \mathrm{m} / \mathrm{ns}(P>1000 \mathrm{GPa})$. As $U_{s}^{\text {diamond }}$ drops from $24.5 \mu \mathrm{m} / \mathrm{ns}$ to $20.2 \mu \mathrm{m} / \mathrm{ns}$ (1000 GPa to $600 \mathrm{GPa}), R_{\lambda=532 n m}$ falls from $30 \%$ to $0.1 \%$ which is the detection limit. The measured insulating-conducting transition region is a little larger than but very close to the predicted solid-liquid coexistence region in VTR $\left(22.0<\mathrm{U}_{\mathrm{s}}<24.6 \mu \mathrm{m} / \mathrm{ns}\right)$. There is a strong correlation between the increase in $\mathrm{R}_{\lambda=532 \mathrm{~nm}}$ and the calculated liquid fraction from VTR as shown by the blue curve in figure 3. Additional evidence for a melting transition is found by studying the release of the shock from the rear surface of the diamond. As seen in figure 1, for shocks below the conducting transition $\left(U_{s}<20 \mu \mathrm{m} / \mathrm{ns}\right.$ or about 550 GPa), we see reflective fringes as the shock exits the free surface. The presence of fringes after shock breakout indicates reflection off a solid surface with strength. In contrast for the higher-pressure shocks, where the diamond shock front is reflective ( $U_{s}>25 \mu \mathrm{m} / \mathrm{ns}$ or about $1000 \mathrm{GPa}$ ), the reflectance abruptly falls to zero upon shock breakout from the diamond-vacuum interface. This behaviour is consistent with freesurface unloading of a high-pressure fluid. The unloading fluid rapidly develops a density 
gradient of several tenths of a micron, which is sufficient to extinguish the reflected probe signal. ${ }^{27}$ This observation is strongly indicative of a solid-liquid phase transition occurring in the vicinity of the optical transition.

While the sharp saturation in reflectivity seen in our data at $24.5 \mu \mathrm{m} / \mathrm{ns}$ is consistent with melting to a conducting state, the exponential increase in reflectance with velocity at lower pressure cannot be ascribed entirely to melting. Shock reflectance measurements in $\mathrm{LiF}, \mathrm{Al}_{2} \mathrm{O}_{3},{ }^{28}$ and water $^{29}$, have been well matched using a semiconducting (Sommerfeld) model with a small bandgap and a scattering time that meets the Ioffe-Regel criterion ${ }^{30}$. This model assumes that the rise in reflectance is due to thermal excitation of electrons across a mobility gap.

We have modelled our reflectance data using a similar model. Reflectivity is calculated using the Fresnel equations, $R=\mid\left(\sqrt{\varepsilon}-n_{d}\right) /\left(\sqrt{\varepsilon}+n_{d}\right)^{2}$, where $n_{d}=2.424$ is the refractive index of the unshocked diamond. The complex dielectric constant of the shocked material, $\varepsilon$ is calculated from a Drüde formulation such that $\varepsilon=\left(\varepsilon_{b}-\frac{\omega_{p}^{2}}{\omega^{2}(1+i / \omega \tau)}\right)$, where $\varepsilon_{b}=n_{d}^{2}$ represents the bound electron contribution and is assumed to be constant, $\omega_{p}^{2}=\frac{n_{e} e^{2}}{\varepsilon_{0} m_{e}}$ is the plasma frequency, and $\tau$ is the electron scattering time which for this analysis is assumed to be equal to the Ioffe-Regel minimum scattering time. The scattering time is expressed as $\tau=\tau_{\min }=\frac{2}{v_{e}}\left(\frac{3}{4 \pi n_{\text {atom }}}\right)^{1 / 3}$, where $n_{\text {atom }}$ is the atomic density, and $v_{e}$ is the average carrier velocity, which is calculated by integrating over the Fermi distribution at a given temperature. The electron number 
density, $n_{e}=\gamma \int g_{c}(E) f(E, T) d E$ where $\gamma$ is the electron degeneracy, $g_{c}$ is the electronic density of states, and $f$ is the Fermi-Dirac distribution function. The band gap, or mobility gap of the semiconductor enters the analysis through the density of states.

In diamond the temperature at the onset of high shock reflectance is low enough (0.4-0.5 eV) for all EOS's considered, that substantial thermal excitation of free carriers could only occur for a mobility gap far smaller than at ambient pressure. We have therefore modelled the diamond reflectance data using a density dependant mobility gap, $E_{\text {gap }}=E_{0}-A\left(\rho / \rho_{0}-1\right)$ where $E_{0}=5.45 \mathrm{eV}$ is the zero pressure band gap, $A$ is a constant, $\rho$ is the density and $\rho_{0}$ is the zero pressure density ${ }^{28}$. This is consistent with several theoretical predictions that have reported a reduction in $E_{\text {gap }}$ with increasing deviatory stress ${ }^{31-33}$. Fits to our data using this model give $A=6.01 \pm 0.50 \mathrm{eV}$ so that the mobility gap closes (metallization) at $U_{S}=24.3 \pm 2.0 \mu \mathrm{m} / \mathrm{ns}, \rho=6.7 \pm 0.3 \mathrm{~g} / \mathrm{cc}, P=1000 \pm 200$ GPa, and $T=12000 \pm 4000 \mathrm{~K}^{34}$. The large uncertainty in temperature is dominated by the large disagreement in temperatures arising from various treatments of the melt transition by the different equations of state considered here. Fits for a selection of EOS models are shown in figure 3. It is clear that while the analysis matches the low reflectance data quite well, the saturation at about $30 \%$ is much sharper experimentally than can be reproduced solely with this model. Our conclusion is that the best match to the complete data set is provided by a model that combines both a semi-conducting solid with a closing band gap and an eventual transition to a conducting fluid. The diamond-metallic (BC8) solid transition predicted by the KC EOS at 440-500 GPa lies below our observed absorbing to reflecting transition. We see no evidence of a conducting phase in the 300-700 Gpa 
region, although it is possible that the timescale for this transition is slower than our experiment

In summary, laser-driven shock wave experiments have been used to investigate the properties of diamond on its principal Hugoniot between 600 and $3000 \mathrm{GPa}$. Reflectivity measurements show that shock compressed diamond is opaque below 600 GPa, has shock reflectance which increases with pressure between 600 and 1000 GPa, and has nearly constant shock reflectance of about 30\% above $1000 \mathrm{GPa}$. This increase in shock reflectance is caused by a transition to a metallic phase resulting from mobility-gap closure and shock-induced melting. Reflectance measurements of shock front unloading from the free surface are consistent with diamond being solid for $\mathrm{P}<550 \mathrm{GPa}$ and fluid for P>1000 GPa. Our measured optical transition is strongly correlated with the solidliquid coexistence region in the VTR EOS. If the melt transition predicted by RVT is correct, then the ice layers of Neptune and Uranus should contain carbon in the solid phase $^{2}$. 
We thank the operations staff at the Omega laser facility for their efforts during these experiments. This work was performed under the auspices of the U.S. Department of Energy by University of California, Lawrence Livermore National Laboratory under Contract W-7405-Eng-48 


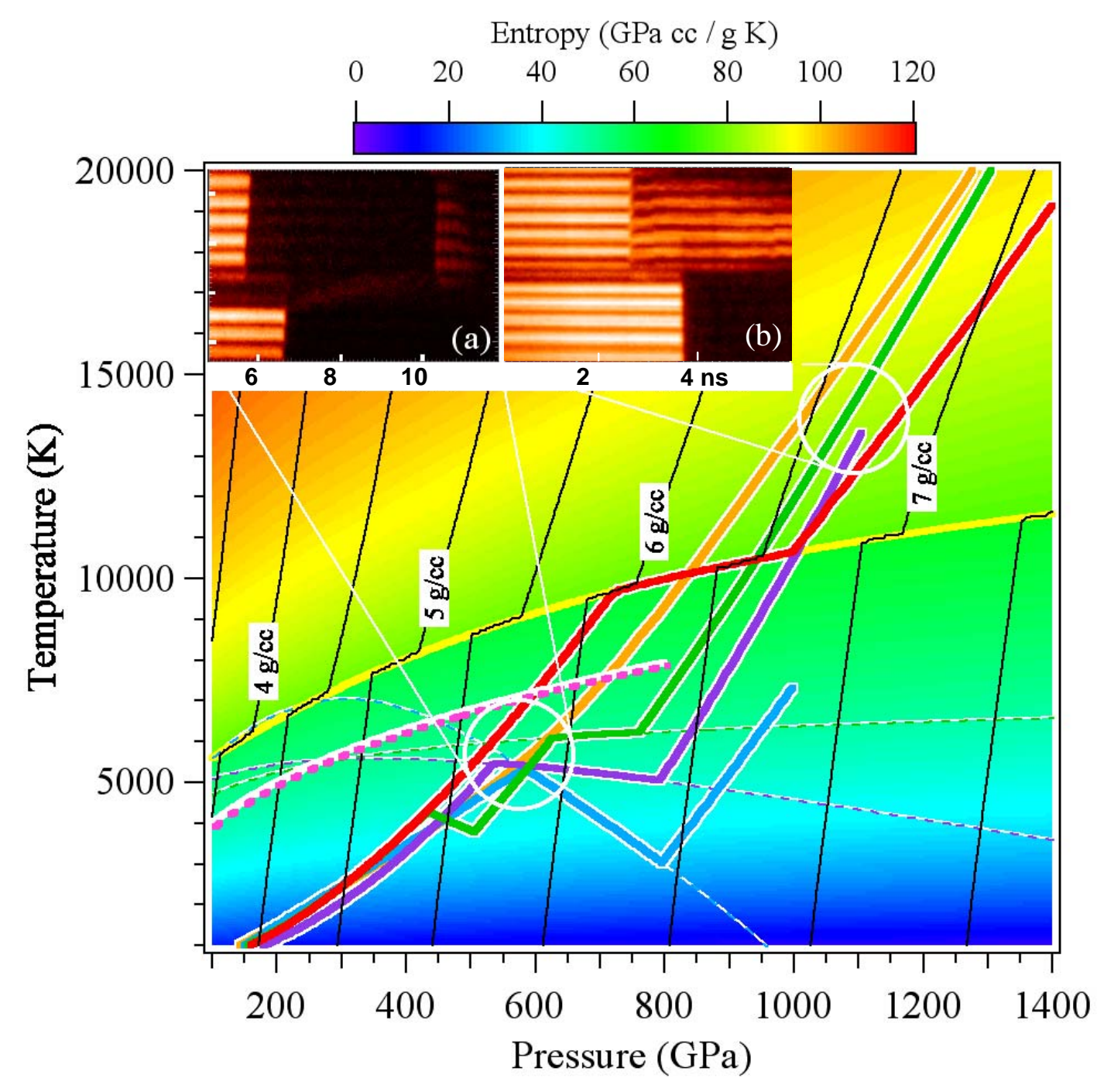

$\begin{array}{ll}\text { Figure } 1 & \text { D. K. Bradley et al }\end{array}$ 


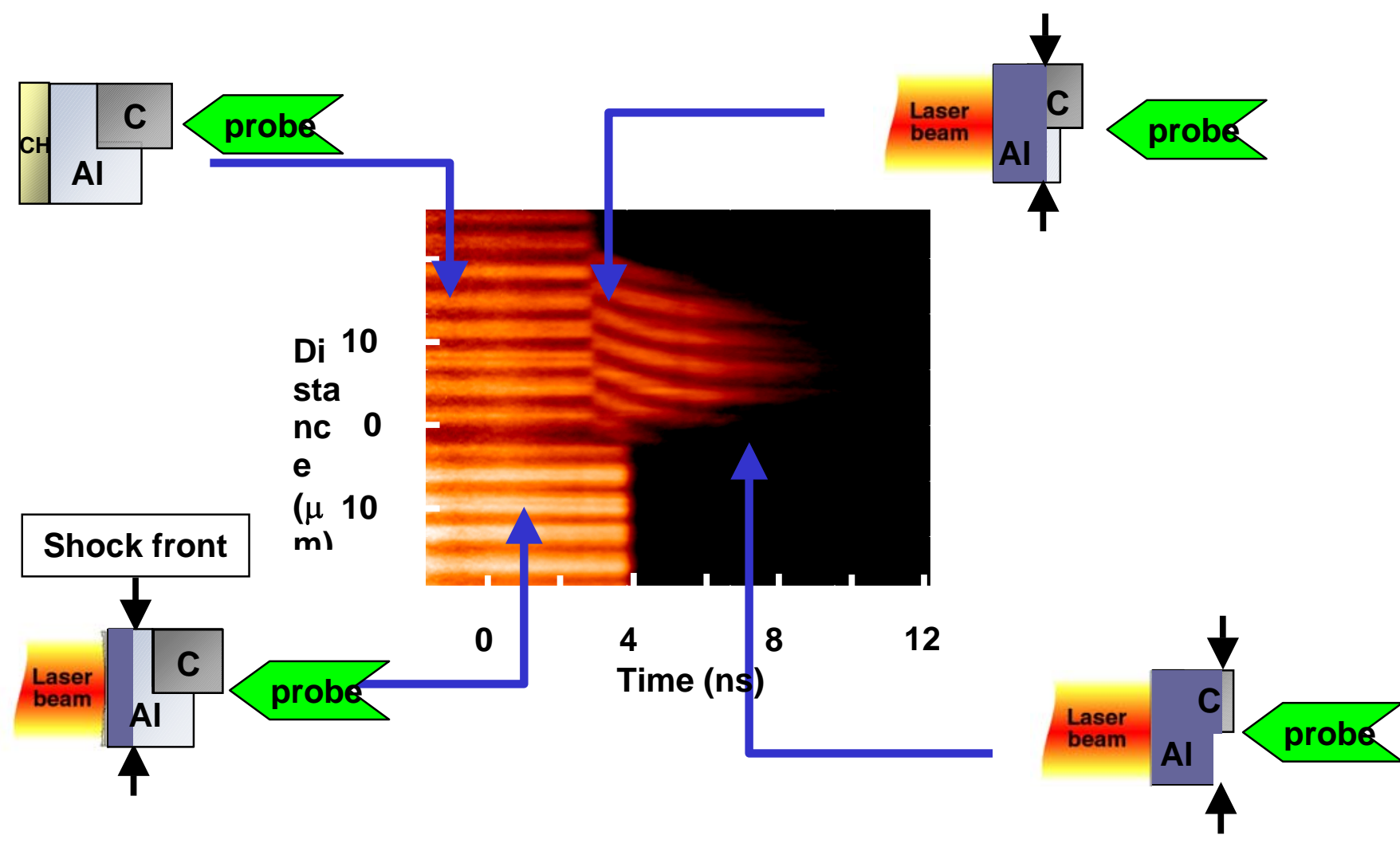

Figure 2 D. K. Bradley et al 


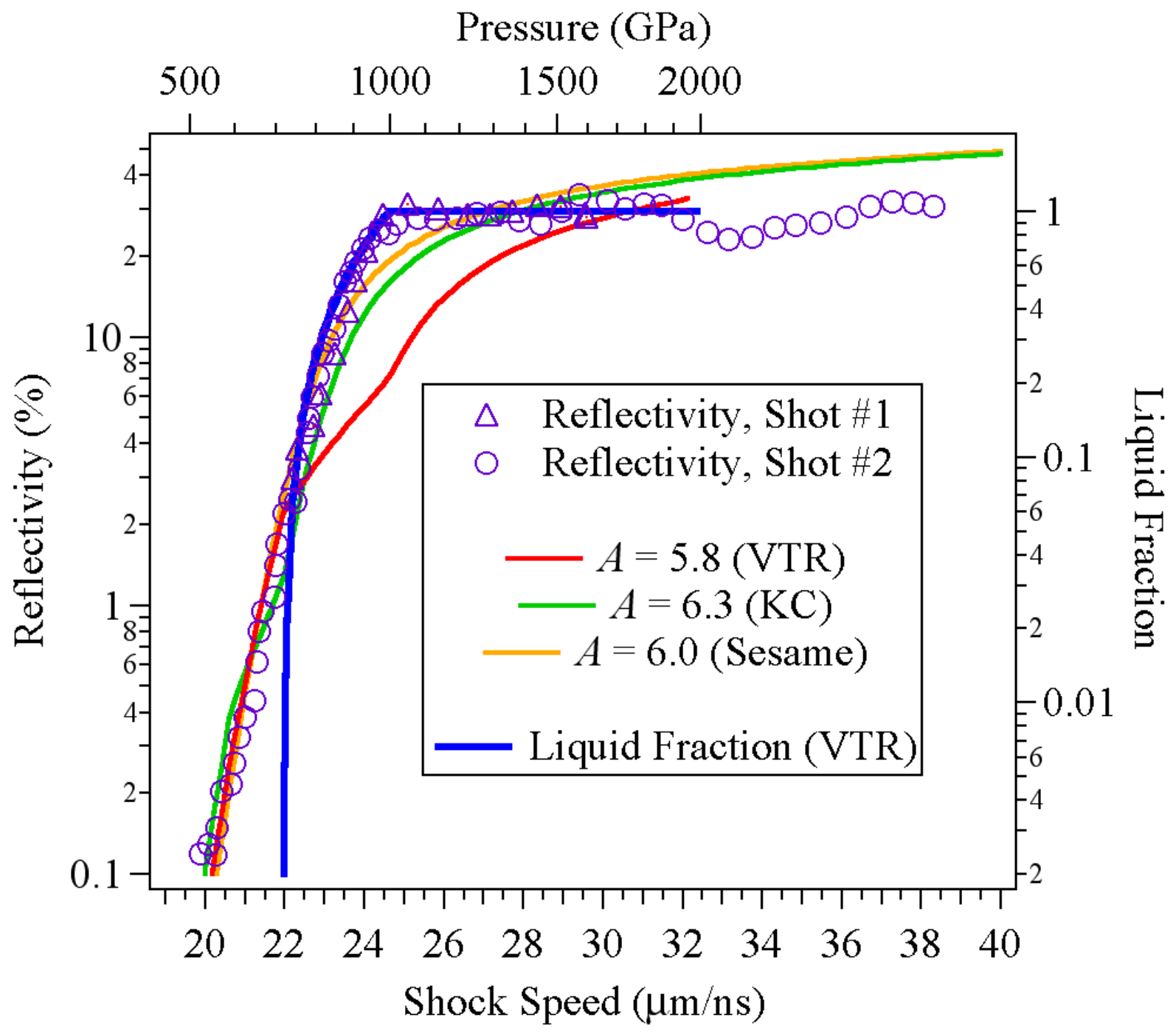

Figure $3 \quad$ D. K. Bradley et al 
FIG. 1. Temperature versus pressure phase diagram for diamond together with the melt (yellow line), Hugoniot (red line), constant densities (black lines), and entropy (color shading) as calculated by VTR ${ }^{9}$. Other Hugoniot predictions include $\mathrm{KC}^{5}$ (green line), Molodets $^{10}$ (purple line), Fried ${ }^{8}$ (blue line) and Sesame ${ }^{17}$ (orange line). The pink dashed line is a calculated isentrope of Neptune. ${ }^{18}$ The inset VISAR images are from diamond shock compressed to a) 550-600GPa and b) 1100 GPa and are discussed in the text. The graphite phase of carbon lies to lower temperatures and pressures than those shown in the figure.

FIG. 2. Typical target and VISAR image for decaying shock used to measure shock reflectance versus shock velocity over the transition region between reflective and nonreflective states. Time zero on this image is set to the beginning of the drive pulse. (a) Before the start of laser drive pulse, unshifted fringes are seen. (b) After the start of drive fringes are still unshifted as shock has not reached rear surface. (c) The shock has broken out into diamond. Shifted fringes are reflected from the shock front in diamond. Unshifted fringes are still seen from thick Al step. (d) The shock has broken out from thick Al step.

FIG. 3. Measured shock reflectance versus shock velocity. The shock pressure axis is calculated from the shock velocity using the VTR EOS ${ }^{9}$, which is limited to pressures below $2000 \mathrm{GPa}$. The solid lines are fits to the Sommerfeld model described in the text using $\gamma=4, \tau=\tau_{\min }$, and $m=m_{e}$. The solid lines shown use the following EOS and values for $A$ : Red line $-\mathrm{VTR}^{9}, A=5.8 \mathrm{eV}$; Green line $-\mathrm{KC}^{5} A=6.3 \mathrm{eV}$; Orange lineSesame $^{17}, A=6.0 \mathrm{eV}$. The blue dotted line is the liquid volume fraction using the VTR EOS. 
W. B. Hubbard, Science 214, 145 (1981).

M. Ross, Nature 292, 435 (1981).

P. Loubeyre, F. Occelli, and R. LeToullec, Nature 416, 613 (2002).

R. Grover, Journal of Chemical Physics 71, 3824 (1979).

G. I. Kerley and L. Chhabildas,, (Sandia National Laboratories, Albuquerque, NM, 2001).

M. P. Grumbach and R. M. Martin, Physical Review B-Condensed Matter 54, R15730 (1996).

D. A. Young and R. Grover, in Shock Waves in Condensed Matter 1987.

Proceedings of the American Physical Society Topical Conference. North-

Holland. 1988, pp.131-4. Amsterdam, Netherlands., edited by S. C. Schmidt and N. C. Holmes.

L. E. Fried and W. M. Howard, Physical Review B 61, 8734 (2000).

M. van Thiel and F. H. Ree, High Pressure Research 10, 607 (1992).

A. M. Molodets, M. A. Molodets, and S. S. Nabatov, in Shock Compression of Condensed Matter, edited by Schmidt, Dandekar and Forbes (AIP Press, 1998), Vol. 429, p. 91.

J. W. Shaner, J. M. Brown, C. A. Swenson, et al., in Journal de Physique (Paris), Colloque, vol.45, no.C-8, Nov. 1984, pp.235-7. France., 1984), Vol. 45, no. C8, p. 235.

F. P. Bundy, W. A. Bassett, M. S. Weathers, et al., Carbon 34, 141 (1996).

J. A. Van Vechten, Physical Review B (Solid State) 7, 1479 (1973). 
F. P. Bundy, Journal of Chemical Physics 38, 618 (1963).

N. S. Fateeva and L. F. Vereshchagin, Pis'Ma V Zhurnal Eksperimental'Noi i

Teoreticheskoi Fiziki 13, 157 (1971).

M. Togaya, Physical Review Letters 79, 2474 (1997).

S. P. Lyon and J. D. Johnson,, (Los Alamos National Laboratory, 1992).

W. B. Hubbard, M. Podolak, and D. J. Stevenson, Neptune and triton. Univ. Arizona Press. 1995, 109.

S. Fahy and S. G. Louie, Physical Review B-Condensed Matter 36, 3373 (1987).

D. K. Bradley, T. R. Boehly, D. L. Brown, et al., in Laser Interaction and Related Plasma Phenomena, edited by H. Hora and G. H. Miley (Plenum Press, New York, 1991), Vol. 7, p. 323.

P. M. Celliers, G. W. Collins, L. B. Dasilva, et al., Applied Physics Letters 73, 1320 (1998).

Strong shocks ( $\mathrm{P}>7-8 \mathrm{Mbar})$ in diamond reflect the probe light directly off of the shock front allowing measurement of $\mathrm{R}_{\lambda=532 \mathrm{~nm}}$ and $u_{s}^{\text {diamond }}$. The Doppler shift of the reflected light results in a fringe shift $\phi$, at the output of the interferometer. When the reflecting surface is the shock front, $u_{s}^{\text {diamond }}=\phi \lambda /\left[4 \tau n_{d}(1+\delta)\right]$, where $\lambda=532 \mathrm{~nm}$ is the laser wavelength in vacuum, $n_{d}=2.424$ is the refractive index of the unshocked diamond, $\delta$ is a wavelength dependent correction due to dispersion in the etalon and $\tau=\left(n_{\text {etalon }}-1 / n_{\text {etalon }}\right) d / c$ is the time delay in the velocity interferometer where $c$ is the speed of light, and $d$ and $n_{\text {etalon }}$ are the thickness and refractive index of the etalon respectively. 
Uncertainty in $u_{s}^{\text {diamond }}(<5 \%)$ is set by the uncertainty in $\phi$ since $\lambda, n_{d}$ and $\tau$ are determined to a precision of 4 significant figures. (1993).

P. Celliers and A. Ng, Physical Review A 47, 3547 (1993).

P. M. Celliers, G. W. Collins, L. B. Da Silva, et al., Physical Review Letters 84, 5564 (2000). City, CA, 1988). D. G. Hicks, P. M. Celliers, G. W. Collins, et al., Physical Review Letters 91, 035502/1 (2003).

P. M. Celliers et al. "Equation of state and optical properties of water compressed by strong shock waves”, submitted to Physics of Plasmas S. B. Kormer, Soviet Physics Uspekhi 11, 229 (1968). M. P. Surh, S. G. Louie, and M. L. Cohen, Physical Review B-Condensed Matter 45, 8239 (1992). H. K. Mao and R. J. Hemley, Nature 351, 721 (1991). O. H. Nielsen, Physical Review B-Condensed Matter 34, 5808 (1986). We have calculated the stated uncertainties by examining several assumptions in our model including the band degeneracy $\gamma$, the relaxation time $\tau \equiv \tau_{\min }$, and the effective carrier mass $m \equiv m_{e}$, as well as the equation of state used to calculate density, and temperature from the measured shock speed. We report average values and standard deviations of the results for several fits using $1 \leq \gamma \leq 4$, 
$\tau_{\min } \leq \tau \leq 2 \tau_{\min }$, and $0.5 m_{e} \leq m \leq 2 m_{e}$ for each of three equations of state, VTR, KC, and Sesame. While varying these parameters affects the magnitude of the reflectivity after saturation and the width of the transition, it does not change the key result: the mobility (band) gap must close at or near the reflecting transition. It is impossible to get the high reflectivity we observe by thermal excitation over a large mobility gap 